$517.5,517.9$

doi: $10.13108 / 2017-9-1-89$

UDC $517.5,517.9$

\title{
SHARP HARDY TYPE INEQUALITIES WITH WEIGHTS DEPENDING ON BESSEL FUNCTION
}

\author{
R.G. NASIBULLIN
}

\begin{abstract}
We prove exact Hardy type inequalities with the weights depending on a Bessel function. We obtain one-dimensional $L^{p}$-inequalities and provide an example of extending these inequalities for the case of convex domains with a finite inner radius. The proved statements are generalization for the case of arbitrary $p \geqslant 2$ of the corresponding inequality proved by F.G. Avkhadiev and K.-J. Wirths for $p=2$.
\end{abstract}

Keywords: Hardy inequality, Bessel function, Lamb constant, distance function, inner radius, convex domains.

Mathematics Subject Classification: 26D15

\section{INTRODUCTION}

Hardy type inequalities relate a function and its derivative in an integral relation and these inequalities are a tool for solving some problems of the mathematics and mathematical physics. Hardy inequalities with arbitrary weights were studied systematically in works by B. Levin [1], P.R. Beesack [2], G. Talenti [3], G. Tomaselli [4], B. Muckenhoupt [5], G. Sinnamon and V.D. Stepanov [6] and by other mathematicians. For instance, G. Talenti and G. Tomaselli obtained necessary and sufficient conditions for the weight functions, for which the corresponding inequalities hold true. We also mention work [7] by F.G. Avkhadiev and K.-J. Wirths, in which they established Hardy type inequalities with weight functions depending of the Bessel function of order $\nu$ :

$$
J_{\nu}(x)=\sum_{k=0}^{\infty} \frac{(-1)^{k} x^{2 k+\nu}}{2^{2 k+\nu} k ! \Gamma(k+1+\nu)}, \quad x>0, \quad \nu \geqslant 0 .
$$

Let us formulate this result.

Theorem A. Let $s \in(0,+\infty), \nu \in(0,+\infty), q>0, \Phi_{\nu, q}(t):=\sqrt{x} J_{\nu}\left(\lambda(2 / q) t^{q / 2}\right)$ and $u:[0,1] \rightarrow \mathbb{R}$ be an absolutely continuous function such that $u(0)=0$ and $u^{\prime} / t^{(s-1)(1+q \nu) / 4} \in$ $L^{2}[0,1]$. Then

$$
\int_{0}^{1} u^{\prime 2} \frac{d t}{\Phi_{q, \nu}^{s-1}(t)} \geqslant s \int_{0}^{1} \frac{u^{2}(t)}{t^{2}}\left(\frac{1-\nu^{2} q^{2}}{4}+\frac{q^{2} \lambda_{\nu}^{2}(2 / q)}{4 t^{-q}}\right) \frac{d t}{\Phi_{q, \nu}^{s-1}(t)} .
$$

The inequality is sharp if $f \not \equiv 0$ and $s \leqslant \frac{1-\nu q}{1+\nu q}$. If $s>\frac{1-\nu q}{1+\nu q}$, then the inequality becomes the identity if and only if $u(t)=C \Phi_{\nu, q}^{s}(t)$, where $C$ is some constant.

R.G. Nasibullin, Sharp Hardy type inequalities with weights DePending on Bessel function.

(C) NAsibullin R.G. 2017.

The work is supported by Russian Foundation for Basic Researches (project no. 14-01-00351-a) and by RFBR and the Government of the Republic of Tatarstan under the project no. 15-41-02433.

Submitted December 8, 2015. 
We note that in paper [7] there were also obtained the analogues of inequality (1) in arbitrary convex domains with a finite inner radius.

Following papers [7] and [8], we call the quantity $\lambda_{\nu}(r)$ defined as a positive root of the equation

$$
r J_{\nu}(z)+2 z J_{\nu}^{\prime}(z)=0
$$

$r>0, \nu \geqslant 0$, the Lamb constant.

Inequality (1) is a logical developing, on one hand, of Hardy type inequalities with weights, and on the other hand, of the inequalities with an additional term. The formulation of problem on obtaining Hardy type inequalities with additional terms is due to H. Brezis and M. Marcus [9]. Brezis and Marcus inequalities were widely developed, for instance, in works [7], [8], [10]16.

This paper is devoted to obtaining an $L^{p}$-analogue of inequality (1). The feature of the obtained inequalities are sharp constants (see, for instance, [7, [8, [10]-[13], [17], [18]) and kernels depending on the Bessel function. It should be noted that by means of the approach by F.G. Avkhadiev (see, for instance, [17], [22]-[24]) each obtained in this paper one-dimensional integral Hardy type inequality can be extended for an arbitrary convex domain $\Omega$ with a finite inner radius

$$
\delta_{0}=\delta_{0}(\Omega)=\sup _{x \in \Omega} \delta
$$

where $\delta=\operatorname{dist}(x, \partial \Omega)$. In order to confirm this possibility, in the paper we provide the following inequality in the spatial case, which can be regarded as one of the main results of the paper.

Let $C_{0}^{1}(\Omega)$ be a family of continuously differentiable functions $f$ with compact supports in $\Omega$. The following theorem holds.

Theorem 1. Let $\Omega$ be a n-dimensional convex domain in the Euclidean space $\mathbb{R}^{n}$, $\delta_{0}=$ $\delta_{0}(\Omega)<\infty$. If $s \in(0,+\infty), \nu \in(0,+\infty), p \in[2,+\infty)$, then for an arbitrary function $f \in C_{0}^{1}(\Omega)$ the following Hardy type inequality

$$
\int_{\Omega} \frac{|\nabla f(x)|^{p}}{\sin ^{s-1}\left(\frac{\pi \delta}{2 \delta_{0}}\right)} d x \geqslant \frac{(p+s-2)^{p-1}}{(p-1)^{p-2}} \frac{\pi^{2}}{\left(2 \delta_{0}\right)^{p}} \int_{\Omega} \frac{|f(x)|^{p}}{\sin ^{s-1}\left(\frac{\pi \delta}{2 \delta_{0}}\right)}\left(\cot \frac{\pi \delta}{2 \delta_{0}}\right)^{p-2} d x
$$

holds true, where $j_{\nu-1}$ is the first positive zero of the Bessel function $J_{\nu-1}(x)$.

Particular cases of this result are related with Poincaré inequality proved by J. Hersch in [19] and by L.E. Payne and I. Stakgold in [20].

\section{AuXiliary ReSUlts}

We shall need some properties of the Bessel function. In paper [7] the authors introduced the function

$$
F_{\nu, r, q}(t)=t^{r / 2} J_{\nu}\left(\lambda_{\nu}(2 r / q) t^{q / 2}\right), \quad t \in[0,1],
$$

where $J_{\nu}$ stands for the Bessel function.

It is known [8] that the Lamb constant $\lambda_{\nu}$ is related with the first positive root $j_{\nu}$ of the Bessel function $J_{\nu}$ of order $\nu$ as follows

$$
\lambda_{\nu}(2 \nu)=j_{\nu-1}
$$

We observe that the function $y=F_{\nu, r, q}(t)$ is the solution to the following differential equation

$$
t^{2} y^{\prime \prime}+(1-r) t y^{\prime}+\left(\frac{r^{2}-\nu^{2} q^{2}}{4}+\frac{q^{2} \lambda_{\nu}^{2}(2 r / q)}{4 t^{-q}}\right) y=0
$$

Let

$$
F_{\nu}(t):=F_{\nu, 1, q}(t) \quad \text { as } \quad \nu=1 / q \text {. }
$$


Employing relation (2) and the behavior of the Bessel function at zero, namely,

$$
J_{\nu}(t)=\frac{1}{\Gamma(1+\nu)}\left(\frac{t}{2}\right)^{\nu}+o(t), \quad \text { as } \quad t \rightarrow 0,
$$

we obtain easily that

$$
F_{\nu}(t)=\sqrt{t} J_{\nu}\left(j_{\nu-1} t^{1 /(2 \nu)}\right)=\frac{j_{\nu-1}^{\nu}}{2^{\nu} \Gamma(1+\nu)} t+o(t), \quad t \rightarrow 0+.
$$

In paper [10] there were provided the following properties of the function $F_{\nu}$ :

$$
F_{\nu}^{\prime}(1)=0, \quad F_{\nu}(t)>0, \quad x \in(0,1] \quad \text { and } \quad F_{\nu}^{\prime}(t)>0, \quad t \in(0,1) .
$$

For an absolutely continuous function $u$ such that $u(0)=0$ and $t^{(1-s) / p} u^{\prime} \in L^{p}(0,1)$, by the inequality $|u(x)| \leqslant \int_{0}^{x}|u(t)| d t$ and by the Hölder inequality we get

$$
\begin{aligned}
|u(x)|^{p} & \leqslant\left(\int_{0}^{x}\left|u^{\prime}(t)\right| d t\right)^{p}=\left(\int_{0}^{x} t^{\frac{s-1}{p-1}} d t\right)^{p-1} \int_{0}^{x} \frac{\left|u^{\prime}(t)\right|^{p}}{t^{s-1}} d t \\
& =\left(\frac{p-1}{s+p-2}\right)^{p-1} x^{s+p-2} \int_{0}^{x} \frac{\left|u^{\prime}(t)\right|^{p}}{t^{s-1}} d t .
\end{aligned}
$$

It is easy to show that

$$
\lim _{t \rightarrow 0} \frac{u^{p}(t) F_{\nu}^{\prime p-1}(t)}{F_{\nu}^{s+p-2}(t)}=0=\frac{u^{p}(1) F_{\nu}^{\prime p-1}(1)}{F_{\nu}^{s+p-2}(1)} .
$$

We shall obtain one-dimensional inequalities as corollaries of D.T. Shum lemma [21], which is formulated as follows.

Lemma B. Let $u(t)$ be an absolutely continuous function on $[a, b]$ such that $u^{\prime}(t) \geqslant 0$ almost everywhere, $Q(t)$ be a positive continuous function on $(a, b), G(u, t)$ be a continuously differentiable function as $t \in[a, b]$ and $u$ is in the range of the function $u(t), G_{u}(u, t)>0$. If the integral is well-defined, then

$$
\int_{a}^{b}\left(Q u^{\prime p}+\left(\frac{c}{p}\right)^{p /(p-1)}(p-1) G_{u}^{p /(p-1)} Q^{-1 /(p-1)}+c G_{t}\right) d t \geqslant c\{G(u(b), b)-G(u(a), a)\},
$$

where $c$ is an arbitrary positive number, $p>1$ and

$$
G_{u}=(\partial / \partial u) G(u, x), \quad G_{x}=(\partial / \partial x) G(u, x) .
$$

This inequality becomes the identity if and only if the following differential equation

$$
u^{\prime}=\left(\frac{c}{p}\right)^{1 /(p-1)}\left(\frac{G_{u}}{Q}\right)^{1 /(p-1)}
$$

is satisfied.

Remark 1. It should be mentioned that the statement of Lemma B implies immediately the inequalities only for monotone functions. The following arguing show that the corresponding Hardy inequality for monotone functions implies the result for arbitrary functions.

Assume that a monotone positive function $g$ and positive weight functions $w$ and $v$ satisfy the following inequality:

$$
\int_{a}^{b} g^{p}(x) w(x) d x \leqslant C_{1} \int_{a}^{b} g^{\prime p}(x) v(x) d x
$$


We let

$$
g(x)=\int_{0}^{x}\left|f^{\prime}(t)\right| d t \quad \text { and } \quad f(x)=\int_{0}^{x} f^{\prime}(t) d t
$$

Then

$$
|f(x)| \leqslant \int_{0}^{x}\left|f^{\prime}(t)\right| d t=g(x), \quad g^{\prime}(x)=\left|f^{\prime}(x)\right|
$$

This implies that

$$
\int_{a}^{b}|f(x)|^{p} w(x) d x \leqslant \int_{a}^{b} g^{p}(x) w(x) d x \leqslant C_{1} \int_{a}^{b}\left(g^{\prime}(x)\right)^{p} v(x) d x=C_{1} \int_{a}^{b}\left|f^{\prime}(x)\right|^{p} v(x) d x .
$$

Thus, we obtain the inequality for an absolutely continuous function.

It is clear that if at some function $g_{0}$ inequality (5) becomes the identity, then in the class of absolutely continuous functions the constant in the inequality is sharp.

\section{MAIN RESULTS ON ONE-DIMENSIONAL INTEGRALS}

We succeeded to find particular cases of the functions $G$ and $Q$, for which by means of the properties of the Bessel function and Lemma B we can obtain the following statement.

Theorem 2. Let $s \in(0,+\infty), \nu \in(0,+\infty), p \in[2,+\infty)$ and $F_{\nu}(t)=\sqrt{t} J_{\nu}\left(j_{\nu-1} t^{1 /(2 \nu)}\right)$. If a function $u:[0,1] \rightarrow \mathbb{R}$ is absolutely continuous, $u(0)=0$ and $u^{\prime} / t^{(s-1) / p} \in L^{p}(0,1)$, then

$$
\int_{0}^{1}\left|u^{\prime}(t)\right|^{p} \frac{d t}{F_{\nu}^{s-1}(t)} \geqslant \frac{(p+s-2)^{p-1}}{(p-1)^{p-2}} \frac{j_{\nu-1}^{2}}{4 \nu^{2}} \int_{0}^{1} \frac{|u(t)|^{p}}{t^{2-\frac{1}{\nu}}}\left(\frac{F_{\nu}^{\prime}(t)}{F_{\nu}(t)}\right)^{p-2} \frac{d t}{F_{\nu}^{s-1}(t)} .
$$

The inequality becomes the identity if and only if

$$
u(t)=C F_{\nu}^{\frac{p+s-2}{p-1}}(t)
$$

where $C$ is some constant.

Proof of Theorem 2. Without loss of generality, it is sufficient to prove the theorem for positive and monotone functions, since for arbitrary functions the inequalities are implied as corollaries.

In Lemma $\mathrm{B}$ we let $a=\varepsilon, b=1$ and

$$
c=\left(\frac{p+s-2}{p-1}\right)^{p-1}, \quad G(u, t)=\frac{u^{p}}{F_{\nu}^{s-1}(t)}\left(\frac{F_{\nu}^{\prime}(t)}{F_{\nu}(t)}\right)^{p-1}, \quad Q(t)=\frac{1}{F_{\nu}^{s-1}(t)} .
$$

By simple calculations it is easy to obtain that

$$
\left(\frac{c}{p}\right)^{p /(p-1)}(p-1) G_{u}^{p /(p-1)} Q^{-1 /(p-1)}=c^{p /(p-1)}(p-1) \frac{u^{p}(t)}{F_{\nu}^{s-1}(t)}\left(\frac{F_{\nu}^{\prime}(t)}{F_{\nu}(t)}\right)^{p}
$$

and

$$
c G_{t}=c u^{p}(t)\left(\frac{p-s}{F_{\nu}^{s-1}(t)}\left(\frac{F_{\nu}^{\prime}(t)}{F_{\nu}(t)}\right)^{p}+\frac{(p-1) F_{\nu}^{\prime \prime}(t)}{F_{\nu}^{s}(t)}\left(\frac{F_{\nu}^{\prime}(t)}{F_{\nu}(t)}\right)^{p-2}\right) .
$$


We have

$$
\begin{aligned}
& \left(\frac{c}{p}\right)^{p /(p-1)}(p-1) G_{u}^{p /(p-1)} Q^{-1 /(p-1)}+c G \\
& =\frac{u^{p}(t)}{F_{\nu}^{s-1}(t)}\left(\frac{F_{\nu}^{\prime}(t)}{F_{\nu}(t)}\right)^{p}\left((p-1) c^{p /(p-1)}+c(2-p-s)\right)+c(p-1) u^{p}(t) \frac{F_{\nu}^{\prime \prime}(t)}{F_{\nu}^{s}(t)}\left(\frac{F_{\nu}^{\prime}(t)}{F_{\nu}(t)}\right)^{p-2} \\
& =\left(\frac{p+s-2}{p-1}\right)^{p-1}(p-1) u^{p}(t) \frac{F_{\nu}^{\prime \prime}(t)}{F_{\nu}^{s}(t)}\left(\frac{F_{\nu}^{\prime}(t)}{F_{\nu}(t)}\right)^{p-2} .
\end{aligned}
$$

Hence, it follows from Lemma B that

$$
\begin{aligned}
& \int_{\varepsilon}^{1}\left(\frac{u^{\prime p}(t)}{F_{\nu}^{s-1}(t)}+\left(\frac{p+s-2}{p-1}\right)^{p-1}(p-1) u^{p}(t) \frac{F_{\nu}^{\prime \prime}(t)}{F_{\nu}^{s}(t)}\left(\frac{F_{\nu}^{\prime}(t)}{F_{\nu}(t)}\right)^{p-2}\right) d t \\
& \geqslant\left(\frac{p+s-2}{p-1}\right)^{p-1}\left\{\frac{u^{p}(1)}{F_{\nu}^{s-1}(1)}\left(\frac{F_{\nu}^{\prime}(1)}{F_{\nu}(1)}\right)^{p-1}-\frac{u^{p}(\varepsilon)}{F_{\nu}^{s-1}(\varepsilon)}\left(\frac{F_{\nu}^{\prime}(\varepsilon)}{F_{\nu}(\varepsilon)}\right)^{p-1}\right\} .
\end{aligned}
$$

In the latter inequality we employ the equation

$$
F_{\nu}^{\prime \prime}(t)+\frac{j_{\nu-1}^{2}}{4 \nu^{2}} F_{\nu}(t) t^{-2+\frac{1}{\nu}}=0
$$

and pass to the limit as $\varepsilon \rightarrow 0$. We obtain

$$
\int_{0}^{1} u^{\prime p}(t) \frac{d t}{F_{\nu}^{s-1}(t)} \geqslant \frac{(p+s-2)^{p-1}}{(p-1)^{p-2}} \frac{j_{\nu-1}^{2}}{4 \nu^{2}} \int_{0}^{1} \frac{u^{p}(t)}{t^{2-\frac{1}{\nu}}}\left(\frac{F_{\nu}^{\prime}(t)}{F_{\nu}(t)}\right)^{p-2} \frac{d t}{F_{\nu}^{s-1}(t)} .
$$

Equation (7) is a particular case of (3) as $\nu=r / q$.

It also follows from Lemma $B$ that the constants are sharp provided

$$
\frac{u^{\prime}(t)}{u(t)}=\frac{p+s-2}{p-1} \frac{F_{\nu}^{\prime}(t)}{F_{\nu}(t)}
$$

That is, as $u(t)=C F_{\nu}^{\frac{p+s-2}{p-1}}(t)$, the inequality becomes the identity. It is easy to check that the function $C F_{\nu}^{\frac{p+s-2}{p-1}}(t)$ satisfies the assumptions of the theorem.

We provide two corollaries of Theorem 2. Employing the identities

$$
J_{1 / 2}(t)=\sqrt{\frac{2}{\pi}} \frac{\sin t}{\sqrt{t}}, \quad J_{-1 / 2}(t)=\sqrt{\frac{2}{\pi}} \frac{\cos t}{\sqrt{t}},
$$

and, as a result, $j_{-1 / 2}=\pi / 2, j_{1 / 2}=\pi$ (see [8] for more details), we obtain

Corollary 1. Let $s \in(0,+\infty), p \in[2,+\infty)$ and an absolutely continuous function $u$ on $[0,1]$ be such that $u(0)=0$ and $\left(u^{\prime}(t)\right)^{p} \sin ^{1-s}(t)$ be integrable over $[0,1]$. Then

$$
\int_{0}^{1} \frac{\left|u^{\prime}(t)\right|^{p}}{\sin ^{s-1}(\pi t / 2)} d t \geqslant \frac{(p+s-2)^{p-1}}{(p-1)^{p-2}}\left(\frac{\pi}{2}\right)^{p} \int_{0}^{1}|u(t)|^{p}\left(\cot \frac{\pi t}{2}\right)^{p-2} \frac{d t}{\sin ^{s-1}(\pi t / 2)} .
$$

The inequality becomes the identity as $u(t)=C \sin ^{\frac{p+s-2}{p-1}}(\pi t / 2)$, where $C$ is some constant.

As $s=1$ and $p=2$ we have a result from [8]. 
Corollary 2. Let $\nu \in(0,+\infty), p \in[2,+\infty)$ and an absolutely continuous function $u$ on $[0,1]$ be such that $u(0)=0$ and $\left(u^{\prime}(t)\right)^{2}$ is integrable over $[a, b]$. Then

$$
\int_{0}^{1}\left|u^{\prime}(t)\right|^{2} \geqslant \frac{j_{\nu-1}^{2}}{4 \nu^{2}} \int_{0}^{1} \frac{|u(t)|^{2}}{t^{2-\frac{1}{\nu}}} d t
$$

The inequality becomes the identity as $u(t)=C \sqrt{t} J_{\nu}\left(j_{\nu-1} \frac{1}{2 \nu}\right)$, where $C$ is a some constant.

The second main result of the paper is also related to a particular case of the function $F_{\nu, r, q}$. We let $\Phi_{q}(t)=F_{0,1, q}(t)$, that is,

$$
\Phi_{q}(t)=\sqrt{t} J_{0}\left(\lambda_{0}(2 / q) t^{q / 2}\right)
$$

The following theorem holds.

Theorem 3. Let $s \in(0,+\infty), \nu \in(0,+\infty), p \in[2,+\infty)$ and an absolutely continuous function $u$ on $[0,1]$ be such that $u(0)=0$ and $u^{\prime} / t^{(s-1) /(2 p)} \in L^{p}[0,1]$. Then

$$
\int_{0}^{1}\left|u^{\prime}(t)\right|^{p} \frac{d t}{\Phi_{q}^{s-1}(t)} \geqslant \frac{(p+s-2)^{p-1}}{(p-1)^{p-2}} \int_{0}^{1}|u(t)|^{p}\left(\frac{1}{4 t^{2}}+\frac{q^{2} \lambda_{0}^{2}(2 / q)}{4 t^{2-q}}\right)\left(\frac{\Phi_{q}^{\prime}(t)}{\Phi_{q}(t)}\right)^{p-2} \frac{d t}{\Phi_{q}^{s-1}(t)} .
$$

As $s>p-1$, the inequality becomes the identity if and only if $u(t)=C\left(\Phi_{q}(t)\right)^{\frac{p+s-2}{p-1}}$, where $C$ is some constant.

Proof of Theorem 3. In Lemma B we let $a=\varepsilon, b=1$ and

$$
c=\left(\frac{p+s-2}{p-1}\right)^{p-1}, \quad G(u, t)=\frac{u^{p}}{\Phi_{q}^{s-1}}\left(\frac{\Phi_{q}^{\prime}(t)}{\Phi_{q}(t)}\right)^{p-1}, \quad Q(t)=\frac{1}{\Phi_{q}^{s-1}(t)} .
$$

Similar to the proof of Theorem 1 we obtain

$$
\begin{gathered}
\int_{\varepsilon}^{1}\left(\frac{u^{\prime p}}{\Phi_{q}^{s-1}(t)}+\left(\frac{p+s-2}{p-1}\right)^{p-1}(p-1) u^{p}(t) \frac{\Phi_{q}^{\prime \prime}(t)}{\Phi_{q}^{s}(t)}\left(\frac{\Phi_{q}^{\prime}(t)}{\Phi_{q}(t)}\right)^{p-2}\right) d t \\
\geqslant c\left(\frac{u^{p}(1)}{\Phi_{q}^{s-1}(1)}\left(\frac{\Phi_{q}^{\prime}(1)}{\Phi_{q}(1)}\right)^{p-1}-\frac{u^{p}(\varepsilon)}{\Phi_{q}^{s-1}(\varepsilon)}\left(\frac{\Phi_{q}^{\prime}(\varepsilon)}{\Phi_{q}(\varepsilon)}\right)^{p-1}\right) .
\end{gathered}
$$

Passing to the limit as $\varepsilon \rightarrow 0$ and employing equation (3) as $\nu=0$, namely,

$$
\Phi_{q}^{\prime \prime}(t)+\left(\frac{1}{4 t^{2}}+\frac{q^{2} \lambda_{0}^{2}(2 / q)}{4 t^{2-q}}\right) \Phi_{q}(t)=0,
$$

we have

$$
\int_{0}^{1} u^{\prime p}(t) \frac{d t}{\Phi_{q}^{s-1}(t)} \geqslant \frac{(p+s-2)^{p-1}}{(p-1)^{p-2}} \int_{0}^{1} u^{p}(t)\left(\frac{1}{4 t^{2}}+\frac{q^{2} \lambda_{0}^{2}(2 / q)}{4 t^{2-q}}\right)\left(\frac{\Phi_{q}^{\prime}(t)}{\Phi_{q}(t)}\right)^{p-2} \frac{d t}{\Phi_{q}^{s-1}(t)} .
$$

Here we have used that

$$
|u(x)|^{p} \leqslant\left(\frac{s+2 p-3}{2(p-1)}\right)^{p-1} x^{(s+2 p-3) / 2} \int_{0}^{x} \frac{\left|u^{\prime}(t)\right|^{p}}{t^{(s-1) / 2}} d t,
$$

and that $\Phi_{q}(t)=O(\sqrt{t})$ for small $t$. 
It also follows from Lemma B that the constants are sharp provided

$$
\frac{u^{\prime}(t)}{u(t)}=\frac{p+s-2}{p-1} \frac{\Phi_{q}(t)}{\Phi_{q}^{\prime}(t)}
$$

That is, as $u(t)=C \Phi_{q}^{\frac{p+s-2}{p-1}}(t)$, the inequality becomes the identity. It is easy to check that as $s>p-1$, the function $\Phi_{q}$ satisfies the assumption of the theorem. At the present, the sharpness as $s<p-1$ is unclear.

The last result of this paper is also related with a particular case of the function $F_{\nu, r, q}$. Let $\Phi_{\nu, q}(t)=F_{\nu, 1, q}(t)$. Then the function

$$
\Phi_{\nu, q}(t)=\sqrt{t} J_{\nu}\left(\lambda_{\nu}(2 / q) t^{q / 2}\right)
$$

satisfies the following differential equation:

$$
t^{2} y^{\prime \prime}+\left(\frac{1-\nu^{2} q^{2}}{4}+\frac{q^{2} \lambda_{\nu}^{2}(2 / q)}{4 t^{-q}}\right) y=0
$$

Arguing as in the proof of Theorem 1, we have

$$
\begin{aligned}
& \int_{\varepsilon}^{1}\left(\frac{u^{\prime p}(t)}{\Phi_{\nu, q}^{s-1}(t)}+\left(\frac{p+s-2}{p-1}\right)^{p-1}(p-1) u^{p}(t) \frac{\Phi_{\nu, q}^{\prime \prime}(x)(t)}{\Phi_{\nu, q}^{s}(t)}\left(\frac{\Phi_{\nu, q}^{\prime}(t)}{\Phi_{\nu, q}(t)}\right)^{p-2}\right) d t \\
& \geqslant c\left(\frac{u^{p}(1)}{\Phi_{q}^{s-1}(1)}\left(\frac{\Phi_{\nu, q}^{\prime}(1)}{\Phi_{q}(1)}\right)^{p-1}-\frac{u^{p}(\varepsilon)}{\Phi_{q, \nu}^{s-1}(\varepsilon)}\left(\frac{\Phi_{\nu, q}^{\prime}(\varepsilon)}{\Phi_{\nu, q}(\varepsilon)}\right)^{p-1}\right) .
\end{aligned}
$$

Passing to the limit and employing equation (11) lead us to the following theorem.

Theorem 4. Let $s \in(0,+\infty), \nu \in(0,+\infty), p \in[2,+\infty)$ and an absolutely continuous function $u$ on $[0,1]$ be such that $u(0)=0$ and $u^{\prime} / t^{(s-1)(1+q \nu) /(2 p)} \in L^{p}[0,1]$. Then

$$
\int_{0}^{1} \frac{\left|u^{\prime}(t)\right|^{p}}{\Phi_{q, \nu}^{s-1}(t)} d t \geqslant \frac{(p+s-2)^{p-1}}{(p-1)^{p-2}} \int_{0}^{1} \frac{|u(t)|^{p}}{t^{2}}\left(\frac{1-\nu^{2} q^{2}}{4}+\frac{q^{2} \lambda_{\nu}^{2}(2 / q)}{4 t^{-q}}\right)\left(\frac{\Phi_{q, \nu}^{\prime}(t)}{\Phi_{q, \nu}(t)}\right)^{p-2} \frac{d t}{\Phi_{q, \nu}^{s-1}(t)} .
$$

As $s>(p-1)\left(\frac{2(p-1)}{1+\nu}-p\right)+1$, the inequality becomes the identity if and only if $u(t)=$ $C\left(\Phi_{q, \nu}(t)\right)^{\frac{p+s-2}{p-1}}$, where $C$ is some constant.

\section{INEQUALITIES IN CONVEX DOMAINS}

Let $\Omega$ be a $n$-dimensional proper subset of the Euclidean space $\mathbb{R}^{n}, \delta=\delta(x)=\operatorname{dist}(x, \partial \Omega)$ and

$$
\delta_{0}=\delta_{0}(\Omega)=\sup _{x \in \Omega} \delta(x)<\infty .
$$

We proceed to proving the main result, Theorem 1. Here we employ the approach by F.G. Avkhadiev, see, for instance, [17], 22]-[24]. We consider two cases of the range of the parameter $n$ : $n=1$ and $n \geqslant 2$. As $n=1$, that is, as $\Omega=(a, b)$, for each continuously differentiable function such that $f(a)=f(b)=0$ we need to prove the inequality

$$
\int_{a}^{b} \frac{\left|f^{\prime}(x)\right|^{p}}{\sin ^{s-1}\left(\frac{\pi \delta}{2 \delta_{0}}\right)} d x \geqslant \frac{(p+s-2)^{p-1}}{(p-1)^{p-2}} \frac{\pi^{p}}{\left(2 \delta_{0}\right)^{p}} \int_{a}^{b}|f(x)|^{p}\left(\cot \frac{\pi \delta}{2 \delta_{0}}\right)^{p-2} \frac{d x}{\sin ^{s-1}\left(\frac{\pi \delta}{2 \delta_{0}}\right)}
$$

where

$$
\delta=\delta(x)=\min \{x-a, b-x\}, \quad \delta_{0}=\frac{b-a}{2} .
$$


For arbitrary $\rho>0$, the change of variable $\tau=\rho t$ in $(9)$ leads us to

$$
\int_{0}^{\rho} \frac{\left|u^{\prime}(\tau)\right|^{p}}{\sin ^{s-1}\left(\frac{\pi \tau}{2 \rho}\right)} d \tau \geqslant \frac{(p+s-2)^{p-1}}{(p-1)^{p-2}} \frac{\pi^{p}}{(2 \rho)^{p}} \int_{0}^{\rho}|u(\tau)|^{p}\left(\cot \frac{\pi \tau}{2 \rho}\right)^{p-2} \frac{d \tau}{\sin ^{s-1}\left(\frac{\pi \tau}{2 \rho}\right)} .
$$

Applying the latter inequality to the functions $u(\tau)=f(\tau+a)$ and $u(\tau)=f(b-\tau)$ with $\rho=\delta_{0}=\frac{b-a}{2}$, we get

$$
\begin{aligned}
& \int_{a}^{(a+b) / 2} \frac{\left|f^{\prime}(x)\right|^{p}}{\sin ^{s-1}\left(\frac{\pi(x-a)}{2 \rho}\right)} d x \geqslant \frac{(p+s-2)^{p-1}}{(p-1)^{p-2}} \frac{\pi^{p}}{\left(2 \delta_{0}\right)^{p}} \int_{a}^{(a+b) / 2}|f(x)|^{p}\left(\cot \frac{\pi(x-a)}{2 \delta_{0}}\right)^{p-2} \frac{d x}{\sin ^{s-1}\left(\frac{\pi(x-a)}{2 \rho}\right)} \\
& \text { and } \\
& \int_{(a+b) / 2}^{b} \frac{\left|f^{\prime}(x)\right|^{p}}{\sin ^{s-1}\left(\frac{\pi(b-x)}{2 \rho}\right)} d x \geqslant \frac{(p+s-2)^{p-1}}{(p-1)^{p-2}} \frac{\pi^{p}}{\left(2 \delta_{0}\right)^{p}} \int_{(a+b) / 2}^{b}|f(x)|^{p}\left(\cot \frac{\pi(b-x)}{2 \delta_{0}}\right)^{p-2} \frac{d x}{\sin ^{s-1}\left(\frac{\pi(b-x)}{2 \rho}\right)} .
\end{aligned}
$$

The sum of these two inequalities gives the required statement.

We proceed to the case $n \geqslant 2$. We note that by means of F.G. Avkhadiev method, by onedimensional inequalities we can obtain inequalities in arbitrary even non-convex domains, see, for instance, [17], 22]-24. We give a brief description of this method. Let $\Lambda$ be an arbitrary open domain, in which we need to prove the Hardy type inequality. Approximating the domain $\Lambda$ by cubes, F.G. Avkhadiev showed that it is sufficient to check the inequality on the sets of special form:

$$
K(S)=\left\{x \in \Lambda_{1}: \text { there exists a point } y \in S \text { such that } \delta(x, \Lambda)=|x-y|\right\},
$$

where $\Lambda_{1}$ is a some partition of the domain $\Lambda$ and for $k \in\{1,2, \ldots, n\}, S$ is the $(n-k)$ dimensional cube face.

While calculating the integrals over set $K(S)$, we have to employ either spherical or cylindric or Cartesian coordinates that allows us to pass to the corresponding iterated integral and to prove only one-dimensional inequalities. For a convex domain the situation is simple and onedimensional inequalities are extended straightforwardly to the spatial case. This completes the proof of Theorem 1.

The author thanks Professor F.G. Avkhadiev for valuable advises, comments and various help in all stages of preparing this work.

\section{BIBLIOGRAPHY}

1. V. Levin. Notes on inequalities. II. On a class of integral inequalities // Rec. Math. Moscou. n. Ser. 4. 309-324 (1938). (in Russian).

2. P.R. Beesack. Hardy's inequality and its extensions // Pacific J. Math. 11:1, 39-61 (1961).

3. G. Talenti. Osservazioni sopra una classe di disuguaglianze// Rend. Sem. Mat. Fiz. Milano. 39:1, 171-185 (1969).

4. G. Tomaselli. A class of inequalities // Boll. Un. Mat. Ital. Ser. 2. 21, 622-631 (1969).

5. B. Muckenhoupt. Hardy's inequality with weights // Studia Mathematica. 44:1, 31-38 (1972).

6. G. Sinnamon and V.D. Stepanov. The weighted Hardy inequality: new proofs and the case $p=1$ // J. London Math. Soc. 54:2, 89-101 (1996).

7. F.G. Avkhadiev, K.-J. Wirths. Sharp Hardy-type inequalities with Lamb's constants // Bull. Belg. Math. Soc. Simon Stevin. 18:4, 723-736 (2011).

8. F.G. Avkhadiev, K.-J. Wirths. Unified Poincaré and Hardy inequalities with sharp constants for convex domains // Z. Angew. Math. Mech. 87:8-9, 632-642 (2007).

9. H. Brezis, M. Marcus. Hardy's inequality revisited // Ann. Scuola Norm. Sup. Pisa Cl. Sci. 4. 25:1-2, 217-237 (1997). 
10. F.G. Avkhadiev, K.-J. Wirths. Weighted Hardy inequalities with sharp constants // Lobachevskii J. Math. 31:1, 1-7 (2010).

11. F.G. Avkhadiev and K.-J. Wirths. On the best constants for the Brezis-Marcus inequalities in balls // J. Math. Anal. Appl. 396:2, 473-480 (2012).

12. M. Marcus, V.J. Mizel, Y. Pinchover. On the best constants for Hardy's inequality in $\mathbb{R}^{n} / /$ Trans. Amer. Math. Soc. 350:8, 3237-3250 (1998).

13. M. Hoffmann-Ostenhof, T. Hoffmann-Ostenhof, A. Laptev. A geometrical version of Hardy's inequality // J. Funct. Anal. 189:2, 539-548 (2002).

14. J. Tidblom. A geometrical version of Hardy's inequality for $W_{0}^{1, p}(\Omega) / /$ Proc. Amer. Math. Soc. 132:2, 2265-2271 (2004).

15. S. Filippas, V.G. Maz'ya, A. Tertikas. On a question of Brezis and Marcus // Calc. Var. Part. Diff. Equat. 25:4, 491-501 (2006).

16. R.G. Nasibullin, A.M. Tukhvatullina. Hardy type inequalities with logarithmic and power weights for a special family of non-convex domains // Ufimskij Matem. Zhurn. 5:2, 43-55 (2013). [Ufa Math. J. 5:2, 43-55 (2013).]

17. F.G. Avkhadiev, R.G. Nasibullin. Hardy-type inequalities in arbitrary domains with finite inner radius // Sibir. Matem. Zhurn. 55:2, 239-250 (2014). [Siber. Math. J. 55:2, 191-200 (2014).]

18. R.G. Nasibullin. Sharpness of the constants in logarithmic Hardy type inequalities in open multidimensional domains // Uchenye Zap. Kazan. Gos. Univ. Ser. Fiz.-Matem. Nauki. 5:3, 111-125 (2013). (in Russian).

19. J. Hersch. Sur la fréquence fondamentale d'une membrande vibrante: évaluation par défaut et principe de maximum // Z. Angew. Matem. Phys. 11:5, 387-413 (1960).

20. L.E. Payne and I. Stakgold. On the mean value of the fundamental mode in the fixed membrane problem // Applicable Anal. 3:3, 295-306 (1973).

21. D.T. Shum. On integral inequalities related to Hardy's // Canada. Math. Bull. 14:2, 225-230 (1971).

22. F.G. Avkhadiev. Hardy-type inequalities on planar and spatial open sets // Trudy Matem. Inst. Steklova. 255, 8-18 (2006). [Proc. Steklov Inst. Math. 255, 2-12 (2006).]

23. F.G. Avkhadiev. Hardy type inequalities in higher dimensions with explicit estimate of constants // Lobachevskii J. Math. 21, 3-31 (2006).

24. F.G. Avkhadiev, R.G. Nasibullin, I.K. Shafigullin. Hardy-type inequalities with power and logarithmic weights in domains of the Euclidean space // Izv. VUZov. Matem. 9, 90-94 (2011). [Russ. Math. Izvestiya VUZ. Matem. 55:9, 76-79 (2011).]

Ramil Gaisaevich Nasibullin,

Kazan Federal University,

Lobachevskii Institute of Mathematics and Mechanics,

Kremlevskaya str. 35,

420008, Kazan, Russia

E-mail: nasibullinramil@gmail.com 